\title{
LIE AND JORDAN STRUCTURES IN SIMPLE, ASSOCIATIVE RINGS
}

\author{
I. N. HERSTEIN
}

1. Introduction. In classifying the simple, finite-dimensional Lie algebras over the field of complex numbers, Cartan and Killing showed that, outside of five isolated examples, these fall into four infinite families.

These four infinite families are, roughly speaking, the matrices of trace 0 and the skew-symmetric matrices under some involution defined for the algebra of matrices.

It is natural to ask whether the simplicity of these sets of matrices, as Lie algebras, is really a consequence of the simplicity of the algebra of all $n \times n$ matrices over a field, as an associative ring.

We shall see that this is indeed the case. The simplicity of an associative ring, and by this we mean any simple ring, with no chain conditions assumed (it can even be a radical ring) forces the simplicity or almost-simplicity of certain natural nonassociative structures obtained from the elements and operations of the forementioned associative ring.

However, our prime motivation for undertaking these studies is not the mere desire to generalize these well-known results of Cartan and Killing from the classical case of matrices to arbitrary simple rings. Rather, the foremost reason for investigating these structures is to provide a tool, a set of techniques, an approach, for answering purely associative questions about the associative structure of these simple rings.

As we proceed in the exposition and development of the researches carried out to date we shall, from time to time, point out how the nonassociative theorems proved can be exploited to prove theorems about simple rings; theorems in whose statements and conclusions these nonassociative structures play no role. These applications, we feel, are but the first ones that have been made. We hope that these techniques will be used advantageously more and more to prove other theorems.

Aside from sketching the use of the results in special cases, we shall give no proofs. These can be found in the appropriate references given.

An address delivered before the Chicago meeting of the Society on April 14, 1961 by invitation of the Committee to Select Hour Speakers for Western Sectional Meetings; received by the editors July 20, 1961. 
2. The Lie and Jordan structure of $R$. Given any associative ring $R$ we can render it into a Lie ring by defining, for any two elements $a, b \in R$ a new product, the Lie product, defined by $[a, b]=a b-b a$. Similarly we can make of it a Jordan ring by introducing the Jordan product $a \cdot b=a b+b a$. In characteristic 2 , of course, these two structures coincide.

It is natural to expect that the associative properties of the ring $R$ should reflect heavily in the properties of $R$ as a Lie and a Jordan ring. The influence on these of the assumption that $R$ is a simple ring will be our main concern.

It is appropriate at this point to point out that, in general, in considering such questions as we shall, be they about the appropriate ideal structure, homomorphisms, derivatives, the Jordan situation is much easier to study than the corresponding Lie one. One reason for this is that in the Lie case the center of $R$ constantly gets in our way, so much so, that many questions, completely answered for the Jordan case are virtually untouched in their Lie analogs.

An additive subgroup, $U$, of $R$ will be called a Lie ideal of $R$ if, whenever $u \in U$ and $r \in R$, then $[u, r]=u r-r u \in U$. Similarly it will be called a Jordan ideal of $R$ if, whenever $u \in U$ and $r \in R$, then $u \cdot r=u r+r u \in U$.

Any ordinary, two-sided ideal of $R$ is automatically both a Lie ideal and a Jordan ideal of $R$. The converse, however, need not holdmany examples exist of subsets which are Lie ideals or Jordan ideals but which are not ideals of $R$.

Suppose now that $R$, as an associative ring, is simple; that is, $R$ has no nontrivial two-sided ideals. We restrict $R$ in no other way. The usual procedure for proving theorems about $R$ is to produce a two-sided ideal and then, knowing that this ideal can only be (0) or $R$, we read off the relevant consequences from this knowledge.

Manifestly the definitions of Lie or Jordan ideals are less restrictive than that of an ordinary ideal. Hence they are easier to produce. If we could characterize them tightly, once such an ideal is produced, knowing what possible subsets of $R$ could serve as candidates for such an ideal, we could draw conclusions about the situation at hand from this knowledge. Our aim then becomes, amongst other things, to get such a close characterization of the Lie ideals and of the Jordan ideals of $R$.

For the Jordan structure the situation is extremely clear-cut for we have [9].

THEOREM 1. If $R$ is a simple ring of characteristic different from 2 and 
if $U$ is a Jordan ideal of $R$ then either $U=(0)$ or $U=R$. In other words $R$ is simple as a Jordan ring.

No such simple dichotomy can exist for $R$ as a Lie ring. For, if $Z$ is the center of $R$ then any additive subgroup of $Z$ trivially provides us with a Lie ideal of $R$. At the other end of the spectrum, (if $[A, B]$ denotes the additive subgroup of $R$ generated by all $a b-b a$ for $a \in A$ and $b \in B)[R, R]$ and any additive subgroup of $R$ which contains $[R, R]$ is automatically a Lie ideal of $R$. At best we can only hope to prove a result which will tell us how any given ideal of $R$ is situated relative to these extremes, $Z$ and $[R, R]$. This is accomplished for us by [9].

THEOREM 2. Let $R$ be a simple ring and let $U$ be a Lie ideal of $R$. Then either $U \subset Z$ or $U \supset[R, R]$ except if $R$ is of characteristic 2 and is of dimension 4 over its center $Z$.

The exception is indeed an exception. For, if we take $R$ to be the ring of $2 \times 2$ matrices over a field $F$ of characteristic 2 , the set of all matrices

$$
\left\{\left(\begin{array}{ll}
a & b \\
b & a
\end{array}\right) \mid a, b \in F\right\}
$$

provides us with a Lie ideal of $R$ which neither is in $Z$ nor contains $[R, R]$.

A crucial step en route to establishing Theorem 2 is to prove

THEOREM 3. Let $R$ be a simple ring and suppose that $U$ is both a Lie ideal and an ordinary subring of $R$. Then either $U \subset Z$ or $U=R$ except in the case that $R$ is of characteristic 2 and is 4-dimensional over its center.

We point out this result (Theorem 3) because we shall now show some purely associative results about $R$ which are consequences of it.

If $T$ is a subset of $R$, let $\bar{T}$ denote the subring of $R$ generated by $T$. Using Theorem 3 it is easy to prove

Corollary 1. If $R$ is simple and not a field then $[R, R]=R$.

Corollary 2. If $R$ is simple, $[[R, R],[R, R]]=[R, R]$.

The third, and most interesting corollary, widely generalizes a result of Dieudonné [7]. He proved it for division algebras which are finite-dimensional over their centers. We shall now show it holds for arbitrary simple rings.

Let $R$ be a simple ring of characteristic different from 2 and suppose 
that in $R$ there is defined an involution ${ }^{*}$, that is, a mapping of $R$ into itself satisfying:

$$
\begin{aligned}
a^{* *} & =a, \\
(a+b)^{*} & =a^{*}+b^{*}, \\
(a b)^{*} & =b^{*} a^{*}
\end{aligned}
$$

for all $a, b \in R$.

Let $S=\left\{x \in R \mid x^{*}=x\right\}$ and $K=\left\{x \in R \mid x^{*}=-x\right\} ; S$ is the set of symmetric elements and $K$ that of skew-symmetric elements of $R$ under the involution *. Since the characteristic is not 2 it is trivial that $R=S+K$. In [11] we proved

COROLlaRY 3. If $R$ is a simple ring of characteristic different from 2 and if either $Z=(0)$ or if $R$ is more than 4-dimensional over $Z$ then $\bar{S}=R$.

Proof. By its very definition $\bar{S}$ is a subring of $R$. We claim, in addition, that it is a Lie ideal of $R$. For, if $a \in \bar{S}$ and $s \in S$ then certainly $a s-s a \in \bar{S}$. On the other hand if $a \in \bar{S}$ and $k \in K$ we assert that $a k-k a \in \bar{S}$. To prove this it is enough to prove it for the elements $a \in \bar{S}$ of the form $a=s_{1} s_{2} \cdots s_{n}, s_{i} \in S$. But $a k-k a=\left(s_{1} k-k s_{1}\right) s_{2} \cdots$ $s_{n}+s_{1}\left(s_{2} k-k s_{2}\right) s_{3} \cdots s_{n}+\cdots+s_{1} s_{2} \cdots s_{n-1}\left(s_{n} k-k s_{n}\right)$. However, as is easy to show, if $s \in S, k \in K$ then $s k-k s \in S$. Thus $a k-k a \in \bar{S}$. Since $R=S+K$, we obtain that $[\bar{S}, R] \subset \bar{S}$. That is $\bar{S}$ is a Lie ideal of $R$.

Consequently, by Theorem 3 , either $\bar{S}=R$ or $\bar{S} \subset Z$. The first possibility is the desired conclusion. Suppose, then, that the second one prevails. Then certainly $S \subset Z$. In particuar, if $k \in K$ since $k^{2} \in S$, we have that $k^{2} \in Z$. Now if $r \in R, r=s+k$, where $s \in S \subset Z$, and $k \in K$, whence $(r-s)^{2}=k^{2} \in Z$, and so $r^{2}-2 r s+s^{2}-k^{2}=0$. Therefore every element in $R$ satisfies a quadratic equation over $Z$. Using standard results from the Jacobson structure theory of primitive rings (see, for instance, [17]) it can be shown that $R$ is at most 4-dimensional over $Z$.

Here, too, the exception is a true exception. If one takes the division ring of quaternions over the real field with its usual ${ }^{*}$ the symmetric elements are merely the reals and they certainly do not generate the whole ring.

Of much greater difficulty and depth is the fact that if $R$ is more than 4-dimensional over $Z$ then $\bar{K}=R$. More can be said: $K$ can be shown to generate $R$ in a very specific and nice manner. This was done by Baxter [6] in his thesis. We shall return to rings with involution and Baxter's result later.

3. Lie ideal structure of $[R, R]$. Whereas $R$ has some natural Lie ideals, namely the additive subgroups of $Z$ and those containing 
$[R, R]$, in the classical case of matrices over fields it has been known for a long time that $[R, R]$, which in this special situation is nothing but the matrices of trace 0 , except for its intersection with the scalars (which need not be $(0)$ in characteristic $p \neq 0$ ) is a simple Lie algebra.

So, again, let $R$ be any simple ring. An additive subgroup $U$ of $[R, R]$ is called a Lie ideal of $[R, R]$ if $[U,[R, R]] \subset U$.

The key result in this direction is [10].

THEOREM 4. If $R$ is a simple ring and if $U$ is a Lie ideal of $[R, R]$ then either $U=[R, R]$ or $U \subset Z$ except if $R$ is of characteristic 2 and is 4-dimensional over $Z$. In other words, $[R, R] / Z \cap[R, R]$ is a simple Lie ring.

Using Theorem 4 it is an extremely easy matter to prove

THEOREM 5. If $R$ is simple and $U$ is an additive subgroup of $R$ such that $[U,[R, R]] \subset U$ then either $U \subset Z$ or $U \supset[R, R]$ except in the case that $R$ is of characteristic 2 and is 4-dimensional over $Z$.

Alternate proofs of Theorems 4 and 5 have been given by Amitsur [1] and Schenkman [25]. Also special forms of these results for particular classes of rings were obtained by Jacobson and Rickart [19].

Theorem 5, too, enables us to prove a theorem about the associative structure of simple rings. Baxter [5] used it to give a new proof of a result of Hattori [8]. Amitsur [2], about the same time, noticed that the same method will yield a great deal more. His precise result is

THEOREM 6. Let $R$ be a simple ring and let $T$ be a subring of $R$ invariant under all automorphisms of $R$. If $R$ has an idempotent $e \neq 0,1$ then either $T \subset Z$ or $T=R$ except if $R$ is the ring of $2 \times 2$ matrices over the integers mod 2.

Actually, as we shall see in the proof, Amitsur only requires that $T$ is invariant under inner automorphisms. Moreover, one can drop the assumption that $T$ is a subring to that in which it is merely an additive subgroup of $R$; in that case the conclusion reads that $T \subset Z$ or $T \supset[R, R]$.

Hattori's theorem is the special case in which $R$ has descending chain condition (and is not a division ring). Since these always have nontrivial idempotents, Amitsur's result is seen to be a broad generalization of that of Hattori. These results are related to the BrauerCartan-Hua theorem which holds for subdivision rings of division rings [17]. If one should want to set up a Galois theory of general simple rings, a result like Theorem 6 would be absolutely basic. As Amitsur pointed out by an example, one can not prove Theorem 6 for arbitrary simple rings. However, it would be interesting to weaken 
the condition that the ring have an idempotent to something less.

Proof. Suppose that $a \in R$ satisfies $a^{2}=0$. The mapping $\phi: R \rightarrow R$ defined by $\phi(x)=(1+a) x(1-a)$ (the unit element is purely formal) is an automorphism of $R$. Hence, by assumption, $\phi(T) \subset T$. Thus if $t \in T, \phi(t)=t+a t-t a-a t a \in T$. Whence for all $t \in T$ and all $a \in R$ satisfying $a^{2}=0, a t-t a-a t a \in T$. If there is a $\lambda \neq 0,1$ in the centroid of $R$ then $(\lambda a)^{2}=0$, hence $\lambda a t-\lambda t a-\lambda^{2} a t a \in T$. The net result of this is that $\lambda(\lambda-1)(a t-t a) \in T$ (if we assume, as we may, that $T$ is an algebra over the centroid) and so $a t-t a \in T$. Therefore, if the centroid of $R$ is not $G F(2),[T, a] \subset T$ for all elements $a \in R$ such that $a^{2}=0$.

If $a^{2}=b^{2}=0$ and $t \in T$ then $(a b-b a) t-t(a b-b a)=(a t-t a) b$ $-b(a t-t a)+a(b t-t b)-(b t-t b) a$, and since both $a t-t a, b t-t b$ are in $T$ and $a^{2}=b^{2}=0$, by the above, $(a b-b a) t-t(a b-b a) \in T$.

All that remains is to show that in the presence of a nontrivial idempotent in $R$ every element in $[R, R]$ can be written as a sum of elements whose squares are zero and commutators thereof. If this were so, then by the results of the above paragraph $[T,[R, R]] \subset T$ would be a consequence. But then Theorem 5 would tell us that either $T \subset Z$ or $T \supset[R, R]$. In the latter case, since $T$ is a subring of $R$, by Corollary 1 to Theorem 2 we would have that $T=R$. This, of course, would have established the theorem.

All that remains is to show that every element in $[R, R]$ can be written as the sum of elements whose square is zero and of elements of the form $a b-b a$ where $a^{2}=b^{2}=0$.

If $a \in R, a=e a e+e a e^{\prime}+e^{\prime} a e+e^{\prime} a e^{\prime}$ where $e^{\prime}=1-e$. Thus $[R, R]$ is generated by elements of the form [eae,ebe], [eae, ebe'], [eae, e'be], $\left[e^{\prime} a e^{\prime}, e^{\prime} b e^{\prime}\right],\left[e^{\prime} a e^{\prime}, e^{\prime} b e\right],\left[e^{\prime} a e^{\prime}, e b e^{\prime}\right]$. Now $\left[e a e, e b e^{\prime}\right]=e a e b e^{\prime}$ and its square is 0 . We are left with the pieces $e R e$ and $e^{\prime} R e^{\prime}$. Also $R e^{\prime} R=R$, whence $e R e=e R e^{\prime} R e$; that is, eae is a sum of elements of the form $e b e^{\prime} c e$. Thus it is enough to prove the result for elements of the form $\left[e a e^{\prime} b e, e c e\right]$. But $\left[e a e^{\prime} b e, e c e\right]=\left[e a e^{\prime}, e^{\prime} b e c e\right]-\left[e c e b e^{\prime}, e^{\prime} a e\right]$ and each constituent on the right hand side inside the brackets has square 0 . A similar argument works for $e^{\prime} R e^{\prime}$. This completes the proof.

Theorem 6 enables us to prove many theorems about the representations of elements of $R$ in special forms, whenever $R$ has an idempotent, for it is not difficult to construct subrings of $R$ invariant under all automorphisms. To cite one such, the additive subgroup generated by all elements which are right-quasi-regular is invariant under all automorphisms; if the ring has a unit element, the additive subgroup generated by invertible elements also is, etc.

In a slightly different direction Theorem 2 might give us an approach to simple nil rings. Suppose, for instance, that $R$ is a simple 
nil ring with infinite centroid. In $R$ let $T$ be the subring generated by all elements whose square is 0 . Clearly $T$ is invariant under all automorphisms (but of course $R$ no longer has any idempotents). If $a \in R$ then for some integer $n, a^{n}=0$, and so the mapping $x \rightarrow(1-a) x(1-a)^{-1}$ $=(1-a) x\left(1+a+a^{2}+\cdots+a^{n-1}\right)$ is an automorphism of $R$. Thus it takes $T$ into itself. Hence, if $t \in T,-a t+t a-a t a+t a^{2}-a t a^{2}+t a^{3} \ldots$ $\in T$. However, if $\lambda$ is in the centroid of $R,(\lambda a)^{n}=0$ for the same $n$ as is used for $a$. Thus $\lambda(-a t+t a)+\lambda^{2}\left(-a t a+t a^{2}\right) \cdots \in T$. If the centroid is infinite, using a Vandermonde determinant argument we can conclude that $a t-t a$ is in $T$ for all $t \in T$ and all $a \in R$. Thus $T$ is both a Lie ideal and a subring of $R$. By Theorem 2, $T=R$ or $T \subset Z$. Since $R$ is a simple nil ring, $Z=(0)$. Thus we are left with $T=R$. That is any element of $R$ is in a subring generated by a finite number of elements whose square is 0 .

The conjecture is that a simple nil ring must be trivial. However, as we pointed out in [9] to show this it is enough to prove that such a ring is locally nilpotent. When the centroid is infinite the above discussion reduces the problem even further, namely, to proving that the subring generated by a finite number of elements whose square is 0 must be nilpotent. Admittedly, the problem so reduced still looks extremely formidable and difficult, but at least it has been narrowed down to a very special and specific problem. Moreover it affords us a program, an approach, to this important question.

4. Simple rings with involution. So far we have only discussed the extension to arbitrary simple rings of the first family of simple Lie algebras over the complex field, namely the matrices of trace 0 . The other three classes arise from skew matrices under some involution. Our discussion now will have these as a motivating guide.

We now turn to general simple rings $R$ with an involution *. As we did earlier in this paper, for such rings we assume that $R$ is not of characteristic 2 and that $S$ and $K$ denote, respectively, the symmetric and skew-symmetric elements of $R$ under this involution.

A simple check verifies that if $a, b \in S$ then $a b+b a \in S$. Therefore $S$ itself forms a Jordan subring of $R$. One might ask whether $S$, as a Jordan ring, is simple. Similarly it is easy to see that if $a, b \in K$ then $a b-b a \in K$. Hence $K$ is a Lie subring of $R$; likewise $[K, K]$ is a Lie subring of $R$. Here, too, one might ask for a characterization of the Lie ideals of $K$ and of $[K, K]$. These questions are answered completely in $[6 ; 11]$.

Before stating the relevant results, one should point out that this situation should be more difficult than the discussion of the Lie and 
Jordan structure of $R$ or of $[R, R]$. The reason for such an expectation might be that $K$ and $S$ are rather far from being subrings of $R$. Given an element $s \in S$ (or $K$ ) only rarely does as fall back in $S$ (or $K)$. Thus many arguments used in studying $R$ and $[R, R]$ which depended on the fact that both elements $a$ and $x a$ behave in a certain manner no longer can be used. This forces the introduction of different types of techniques which tend to make the discussion more difficult. However, even here, the Jordan results turn out to be significantly simpler than the corresponding Lie results.

There is an additional difficulty that should be pointed out. The $4 \times 4$ matrices, over a field, under the ordinary transpose do not behave well. This must show up somewhere in the proofs. True, the $2 \times 2$ matrices afforded us difficulties before, but that was to be expected since these are as small as a noncommutative simple algebra can possibly be. The $4 \times 4$ matrices are not; thus they should force certain special considerations. They do! This also accounts for the presence in the statements of some theorems of the assumption that the algebra is more than 16-dimensional over its center.

The first result in these directions [11] is about the Jordan structure of $S$ and is precise and decisive. It is

THEOREM 7. If $R$ is a simple ring of characteristic different from 2 with an involution then $S$ is a simple Jordan ring.

As was pointed out earlier, if the center of $R$ is (0) or if $R$ is more than 4-dimensional over its center, $\bar{S}$, the subring generated by $S$, is all of $R$. This was relatively easy as a consequence of the results about the Lie ideal structure of $R$.

Even more important for our purposes, and considerably deeper and more difficult is the following result [11].

THEOREM 8. If the center of $R$ is (0) or if $R$ is more than 4-dimensional over its center, then $\bar{K}$, the subring of the simple ring $R$ generated by $K$, is $R$.

In studying rings with involutions two possibilities arise:

(1) $Z \cap K=(0)$; that is, $\lambda^{*}=\lambda$ for all $\lambda \in Z$. In this case the involution is said to be of the first kind.

(2) $Z \cap K \neq(0)$; that is, there is a $\lambda \neq 0 \in Z$ such that $\lambda^{*}=-\lambda$. Here the involution is said to be of the second kind.

The following general statement can be made: for involutions of the second kind the problems are usually easy, whereas for involutions of the first kind they usually are not. The reason for this is that if $\lambda \in Z$, and $\lambda^{*}=-\lambda \neq 0$ then $K=\lambda S$. Hence, since we know, by 
Theorem 8, a great deal about $S$, we can transfer this information easily to $K$. Unfortunately no such method exists for involutions of the first kind.

As in the case of $R, K$ itself has some natural Lie ideals, namely $Z \cap K$ and $[K, K]$. What we strive to do is to locate any other Lie ideal of $K$ relative to these two.

Our first theorem in this direction [11] is

THEOREM 9. If $R$ is a simple ring with involution of characteristic not 2 and if $R$ is more than 16-dimensional over $Z$ then any Lie ideal, $U$, of $K$ must satisfy either $U \subset Z$ or $U \supset[K, K]$.

We shall not indicate the proof but do single out two steps in this proof.

(1) We show that if $U D[K, K]$ and $U \nsubseteq Z$ is a Lie ideal of $K$ then $U$ must be 3 -dimensional over $Z$.

(2) Using (1) and a series of counting arguments one shows that $R$ is at most 131-dimensional over $Z$.

The latter reduces the problem to finite-dimensional algebras, and hence, to total matrix algebras. Since the bound on the dimension is 131 , we are at worst involved with the $11 \times 11$ matrices. But here the situation is completely described [18] so one can use this description to complete the proof of Theorem 9.

As an immediate corollary of Theorem 9 we can prove

CoRollary. If $R$ is as in Theorem 9 , then $[[K, K],[K, K]]=[K, K]$.

It is natural to investigate $[K, K]$ as a Lie ring. Baxter did this and in [6] he proved

TheOREM 10. If $R$ is as in Theorem 9 and if $U$ is a Lie ideal of $[K, K]$ then either $U \subset Z$ or $U=[K, K]$. That is, $[K, K] / U \cap[K, K]$ is a simple Lie ring.

Using all this information about the Lie ideal structure of $K$ and of $[K, K]$ Baxter then proceeded to prove a theorem about the representation of symmetric elements in simple rings with involutions which even seems to be new for matrices. This result is a purely associative one, whose statement and conclusion do not involve these nonassociative structures. It is

THEOREM 11. Let $R$ be a simple ring of characteristic not 2 with an involution. If $R$ is more than 16-dimensional over $Z$ then every symmetric element in $R$ can be written as a sum of squares of skew-symmetric elements of $R$ with coefficients \pm 1 . 
Thus we see that $S \subset \bar{K}$ in a very particular way; since $K \subset \bar{K}$ and since $R=S+K$, Theorem 11 is seen to be a strong generalization of Theorem 8 .

In [14] we presented an alternate proof of Baxter's theorem. We did so by discussing subsets of $S$ closed under Lie products with $K$ and subsets of $K$ closed under Jordan products with $S$. For these "cross-over" subspaces we proved

THEOREM 12. If $R$ is as in Theorem 11 then

(1) if $U \subset K$ and if $U \circ S=\{u s+s u / u \in U, s \in S\}$ is contained in $U$ then $U=(0)$ or $U=K$.

(2) if $V \subset S$ and $[V,[K, K]] \subset V$ then either $V \subset Z$ or $V \supset[K, S]$.

(Note: In (2) we also require that $R$ is not of characteristic 2 or 3.) Another interesting consequence of Baxter's theorem is the following. Suppose $K$ as a vector space over $Z$ (actually, over $S \cap Z$ ) is finite-dimensional, of dimension $n$. Then $K \circ K$, the additive subgroup of $R$ generated by all $a b+b a, a, b \in K$, is of dimension at most $n^{2}$ over $Z$. But $K \circ K=S$ by Theorem 11 , hence $S$ is of dimension at most $n^{2}$ over $Z$. Since $R=S+K$ this forces $R$ to be finite-dimensional over $Z$, in fact, of dimension at most $n+n^{2}$.

5. Jordan homomorphisms and derivations of prime rings. Running parallel to the study of the ideal structure of the various Lie and Jordan subsystems of a ring $R$ are the problems of the mappings which preserve these structures. Of equal interest are also the analogous questions about their derivations.

Motivated from a quite different direction-certain questions in projective geometry-Ancochea $[3 ; 4]$ initiated investigations of mappings $\phi$ of division algebras finite-dimensional over their centers such that $\phi(a+b)=\phi(a)+\phi(b)$ and $\phi\left(a^{2}\right)=\phi(a)^{2}$ for all $a, b$ in the division algebra. He also assumed the characteristic was not 2. Probing deeper he also determined the nature of such mappings for the ring of all matrices over a field. Hua [16], by making use of a pretty, formal identity involving inverses, extended Ancochea's result to general division rings. Kaplansky [22] redefined these semi-automorphisms to include the case of characteristic 2 by insisting that $\phi(a b a)=\phi(a) \phi(b) \phi(a)$ and showed that the previous results carried over to mappings from one finite-dimensional simple algebra onto another. The results proved usually read that $\phi$ must be an automorphism or anti-automorphism.

Jacobson and Rickart [19] considered such mappings for a special class of primitive rings and obtained some general results; the conclu- 
sions of these usually stated that such mappings were direct sums of automorphisms and anti-automorphisms.

In a paper a few years after these [12] we extended these results to cover arbitrary prime rings - this class includes all primitive and simple rings. However, we assumed that the characteristic was not 2 or 3. Smiley [26] simplified our proof, extended it to cover characteristic 3 and, using Kaplansky's modification of the definition, showed the result then also held in characteristic 2 .

A prime ring $R$ is one in which $a R b=(0)$ implies that $a=0$ or $b=0$. A Jordan homomorphism $\phi$ of one ring, $R$, into another, $R^{\prime}$, is a mapping such that $\phi(a+b)=\phi(a)+\phi(b)$ and $\phi\left(a^{2}\right)=\phi(a)^{2}$ for all $a, b \in R$. In characteristic 2 we demand a little more, namely, that $\phi(a b a)$ $=\phi(a) \phi(b) \phi(a)$. The theorem we proved reads

THEOREM 13. If $\phi$ is a Jordan homomorphism of any ring $R$ onto a prime ring $R^{\prime}$ then $\phi$ is either a homomorphism or an anti-homomorphism.

In particular, for a simple ring $R$ the mappings which preserve its Jordan structure are merely automorphisms or anti-automorphisms of its associative ring structure.

What can one say about the derivations of this structure? Let us recall that a derivation of a ring $R$ is a mapping of $R$ into itself such that $\delta(a+b)=\delta(a)+\delta(b)$ and $\delta(a b)=\delta(a) b+a \delta(b)$ for all $a, b \in R$. Thus a Jordan derivation is an additive mapping $\delta(a \circ b)=\delta(a) \circ b$ $+a \circ \delta(b)$ or, neglecting the effect of characteristic $2, \delta\left(a^{2}\right)=a \delta(a)$ $+\delta(a) a$. We use the latter as our definition of Jordan derivation. In [13] we proved

THEOREM 14. If $\delta$ is a Jordan derivation of a prime ring $R$ then $\delta$ is an ordinary derivation of $R$ except in the case in which $R$ is a commutative integral domain of characteristic 2.

Thus for derivations, too, the (Jordan) derivations of the Jordan structure of a simple, associative ring turn out to be nothing more than those induced by the ordinary derivations of this ring as an associative ring.

For the other natural Jordan subsystem of $R$, namely the set of its symmetric elements $S$ in the case that $R$ has an involution defined on it, Jacobson and Rickart [20] proved that, given a ring $R=A_{n}$ where $n \geqq 3$ if the involution on $R$ is such that for the matrix units $e_{i i}, e_{i i}^{*}=e_{i i}$ and if every symmetric element in $R$ is of the form $r+r^{*}$, then any Jordan homorphism of $S$ can be lifted to an associative homomorphism of $R$ in a unique way. 
In an unpublished piece of work, for general simple rings $R$ with involution, we proved that a Jordan automorphism of the symmetric elements of $R$ can be extended to a mapping $\Phi$ on $[R, R]$ such that $\Phi(a b-b a)=\Phi(a) \Phi(b)-\Phi(b) \Phi(a)$. One would conjecture that any Jordan homomorphism of $S$ can be realized as the restriction of an automorphism of $R$. Although little has been done, in the general case, on Jordan derivations of $S$, here too one might conjecture that these are merely restrictions, to $S$, of ordinary derivations of $R$.

In [12] we generalized the notion of a Jordan homomorphism to that of an $n$-Jordan map, namely an additive mapping, $\phi$, of $R$ into $R^{\prime}$ such that $\phi\left(a^{n}\right)=\phi(a)^{n}$ for all $a \in R$. For these we proved

THEOREM 15. If $\phi$ is an $n$-Jordan homomorphism of $R$ onto the prime ring $R^{\prime}$, which is of characteristic larger than $n$ (or 0 ) and if $R$ has $a$ unit element then $\phi=\epsilon \tau$ where $\tau$ is either a homomorphism or an anti-homomorphism of $R$ onto $R^{\prime}$ and where $\epsilon$ is an $(n-1)$ st root of unity lying in the center of $R^{\prime}$.

It would be a reasonable question to study $n$-Jordan automorphisms of the set of symmetric elements of a simple ring with involution; for odd $n$, such questions about the skew-symmetric elements should also be worthy of investigation. Naturally one could ask the analogous questions about $n$-Jordan derivations of appropriately conditioned prime rings; by such mappings we would mean additive maps $\delta$ such that

$$
\delta\left(a^{n}\right)=\delta(a) a^{n-1}+a \delta(a) a^{n-2}+\cdots+a^{n-1} \delta(a) .
$$

We leave the discussion of Lie mappings to the last section.

6. Possible areas of research. In closing this paper we should like to point out several areas and problems that seem to us to be worthy of some attention:

1. The questions mentioned at the end of the last section about Jordan mappings and derivations of the symmetric elements of a simple ring with involution. Of somewhat lesser interest, but still challenging, are those problems related to the $n$-Jordan automorphisms and derivations, for odd $n$, of the skew-symmetric elements.

2. The very important and interesting problem of finding all the Lie mappings of simple (or, perhaps, even of prime) rings. That is, characterize all additive mapping of a simple ring $R$ into a ring $R^{\prime}$ such that $\psi(a b-b a)=\psi(a) \psi(b)-\psi(b) \psi(a)$. In the case of matrices these are, roughly speaking, of the form $\psi+\tau$ where $\psi$ is either an automorphism or the negative of an anti-automorphism and where $\tau$ is a trace-like mapping into the scalars. 
We would conjecture that a similar result holds for general simple rings. In this full generality little progress has been made. However, as a test case, in characteristic 2 , in a joint paper [15] with Kleinfeld we proved that a Lie mapping of a simple ring which preserves cubes must be an automorphism or an anti-automorphism.

3. The analogous question to that in Problem 2 for Lie derivations, that is, mappings $d$ such that $d[a, b]=[d(a), b]+[a, d(b)]$, would be of interest to investigate. In an unpublished work Kaplansky has shown that if the ring has $n \times n$ matrix units, with $n \geqq 3$, then any Lie derivation is an ordinary one plus an additive map into the center.

4. Problems 2 and 3 in the setting of the simple or almostsimple Lie rings $[R, R]$ and $[R, R] / Z \cap[R, R]$ are worthy of serious attention.

5. To settle the very difficult questions above for the skewsymmetric elements, $K$, of a single ring and their associated simple or almost-simple Lie rings $[K, K]$ and $[K, K] / Z \cap[K, K]$ offers a real challenge. The nature of the $8 \times 8$ matrices over fields under transpose promises interesting side difficulties.

6. In [21], Kaplansky showed that a primitive ring satisfying a polynomial identity was finite-dimensional over its center. In [6], Baxter ran into special rings, with many extra conditions imposed, in which the skew elements satisfied a polynomial identity. In his special case he showed that the ring itself had to satisfy a polynomial identity? Is this true in general? Martindale [23], has shown that a primitive algebraic algebra with involution, whose symmetric elements satisfy a polynomial identity, is finite-dimensional over its center. This lends some support to our conjecture that a simple ring whose symmetric (or skew-symmetric) elements satisfy a polynomial identity is finite-dimensional over its center.

7. If $R$ is a simple ring with an involution of the first kind is $K=[K, K]$ ? For matrices this can be shown using trace arguments; an argument settling the general case might provide techniques that could substitute, in other situations, for the kind of trace arguments used in matrices.

8. One could apply the results on the Lie and Jordan structures to studying more purely associative questions. A few samples:

(a) If $R$ is a simple ring with a nonzero zero-divisor and if $T$ is a subring of $R$ invariant under all the automorphisms of $R$, is $T \subset Z$ or $T=R$ ?

(b) If $R$ is a simple ring, and if the nilpotent elements $R$ form a subring $W$ of $R$, is $W=(0)$ or $W=R$ ? 
(c) In the special case of (b), wherein any two nilpotent elements of $R$ commute is it true that $R$ must have no nilpotent elements?

(d) If $R$ is a simple ring with a nontrivial zero-divisor, is every $a b-b a$ in $R$ a sum of nilpotent elements?

(e) If $R$ is simple and has a nonzero nil right-ideal is $R$ itself nil? This is a special case of the Koethe problem.

9. The study of multiplicative analogs and variants of the Lie question for the group of invertible elements in simple rings with unit elements. See [24].

\section{BIBLIOGRAPHY}

1. S. A. Amitsur, Derivations in simple rings, Proc. London Math. Soc. vol. 7 (1957) pp. 87-112.

2. - Invariant submodules of simple rings, Proc. Amer. Math. Soc. vol. 7 (1956) pp. 987-989.

3. G. Ancochea, Le thêorème de von Staudt en géometrie projective quaternionienne, J. Reine Angew. Math. vol. 184 (1942) pp. 192-198.

4. - On semi-automorphisms of division algebras, Ann. of Math. vol. 48 (1947) pp. 147-153.

5. W. Baxter, Lie simplicity of a special class of associative rings, Proc. Amer. Math. Soc. vol. 7 (1958) pp. 855-863.

6. - Lie simplicity of a special class of associative rings. II, Trans. Amer. Math. Soc. vol. 87 (1958) pp. 63-75.

7. J. Dieudonné, On the structure of unitary groups, Trans. Amer. Math. Soc. vol. 72 (1952) pp. 367-385.

8. A. Hattori, On invariant subrings, Jap. J. Math. vol. 21 (1951) pp. 121-129.

9. I. N. Herstein, On the Lie and Jordan rings of a simple, associative ring, Amer. J. Math. vol. 77 (1955) pp. 279-285.

10. - The Lie ring of a simple, associative ring, Duke Math. J. vol. 22 (1955) pp. 471-476.

11. - Lie and Jordan systems in simple rings with involution, Amer. J. Math. vol. 78 (1956) pp. 629-649.

12. - Jordan homomorphisms, Trans. Amer. Math. Soc. vol. 81 (1956) pp. $331-341$.

13. - Jordan derivations of prime rings, Proc. Amer. Math. Soc. vol. 8 (1957) pp. 1104-1110.

14. - Certain invariant submodules in simple rings with involutions, Duke Math. J. vol. 24 (1957) pp. 357-364.

15. I. N. Herstein and E. Kleinfeld, Lie mappings in characteristic 2, Pacific. J. Math. vol. 10 (1960) pp. 843-852.

16. L. K. Hua, On the automorphisms of a field, Proc. Nat. Acad. Sci. U. S. A. vol. 35 (1949) pp. 386-389.

17. N. Jacobson, Structure of rings, Amer. Math. Soc. Colloquium Publications, vol. $37,1956$.

18. - Classes of restricted Lie algebras of characteristic p. I, Amer. J. Math. vol. 60 (1941) pp. 481-515.

19. N. Jacobson and C. Rickart, Jordan homorphisms of rings, Trans. Amer. Math. Soc. vol. 69 (1950) pp. 479-502. 
20. - Homomorphisms of Jordan rings of self-adjoint elements, Trans. Amer. Math. Soc. vol. 72 (1952) pp. 310-322.

21. I. Kaplansky, Rings with polynomial identity, Bull. Amer. Math. Soc. vol. 54 (1948) pp. 575-580. 527.

22. - Semi-automorphisms of rings, Duke Math. J. vol. 14 (1947) pp. 521-

23. W. Martindale, Primitive algebraic algebras with involution, to appear.

24. A. Rosenberg, The structure of the infinite general linear group, Ann. of Math. vol. 68 (1958) pp. 278-294.

25. E. Schenkman, On a theorem of Herstein, Proc. Amer. Math. Soc. vol. 10 (1959) pp. 87-112.

26. M. F. Smiley, Jordan homomorphisms onto prime rings, Trans. Amer. Math. Soc. vol. 84 (1957) pp. 426-429.

\section{CORNELl UNIVERSITY AND}

The University of Chicago 